marked fluctuations in clinical manifestations and its natural history is not yet clear. The validity of comparing clinical measures such as weight gain and numbers of infectious episodes before and after an intervention is therefore highly questionable. Intervention is likely to begin at a time when a child is sicker than usual or has recently suffered an increase in symptoms and so by chance alone there are likely to be fewer recurring symptoms after the intervention. In addition the number of infections experienced by very young children (especially those living in deprived circumstances) is likely to decrease with increasing age.

Other reports of the use of intravenous gammaglobulin in children with symptomatic HIV infection have similarly been small, uncontrolled and retrospective. ${ }^{2-5}$ The lack of a controlled trial has resulted in a diversity of clinical practice concerning its use. The theoretical advantages are that it may decrease numbers of bacterial infections and provide passive immunity to children whose antibody response to antigenic stimuli may be poor. However, the not inconsiderable disadvantages include the risk of acquiring hepatitis $\mathrm{C}$, the theoretical possibility of increasing HIV activation via antigenic stimulation, the psychological trauma and pain of giving intravenous infusions every three to four weeks to young infants, and finally the cost of treatment.

A recent survey of 32 centres in the USA including four AIDS clinical trial units found that of 95 severely ill children, $62 \%$ were receiving neither prophylactic antibiotics nor intravenous immunoglobulin and of 341 symptomatic, but less severely ill children, $84 \%$ received neither treatment. These results reflect the belief of many investigators that routine treatment with intravenous immunoglobulin should not be recommended without proof of efficacy. ${ }^{6}$ There are, of course, a small group of HIV infected children such as those with hypogammaglobulinaemia where this treatment may be beneficial.

There is, at present, a placebo control trial comparing intravenous immunoglobulin with intravenous albumin as placebo being conducted in the USA. Two hundred and thirty eight children from 25 centres in the USA have been recruited for the study and results are expected in the early $1990 \mathrm{~s}^{7}$ Until these are available no definite recommendations about the use of intravenous immunoglobulin in HIV infected children can be made, and its indiscriminate use should be discouraged.

D GIBB

Department of Infectious Diseases, Hospital for Sick Children, Great Ormond Street, Great Ormond Street,
London WCIN $3 f H$

1 Hague RA, Yap PL, Mok JYQ, et al. Intravenous immunoglobulin in HIV infection: evidence for the efficacy of treatment. Arch Dis Child 1989;64:1146-50

2 Oleske JM, Connor EM, Bohila R, et al. The use of IV IgG in children with AIDS. Vox Sang 1987:52:162-75.
3 Schaad UB, Gianella-Borradori A, Perret B, et al. Intravenous immune globulin in symptomatic paediatric human immuno-deficiency virus infection. Eur $\mathcal{f}$ Pediatr 1988;147:300-3.

4 Calvelli TA, Rubinstein A. Intravenous gammaglobulin in infant acquired immunodeficiency syndrome. Peditar Infect Dis 1986;5:S207-10. Gupta A, Novick B, Rubinstein A. Restoration of suppressor $\mathrm{T}$-cell functions in children with AIDS following intravenous gamma globulin treatment. Am f Dis Child 1986;140:143-6.

6 Nicholas SW, Sondheimer DL, Willoughby AD, et al. Human immunodeficiency virus infection in childhood, adolescence, and pregnancy: a status report and national research agenda. Pediatrics 1989;83:293-308.

7 Riqau-Pérez JG (for the IVIG Clinical Trial Study Group, NIH Bethesda, Maryland, USA). Compliance with an intravenous placebo in a randomised trial of intravenous immunoglobulin in HIV-infected children. Implications of AIDS for Mother and Child, Paris, Nov 1989. Abstract H3:177.

Drs Mok and Yap comment:

Dr Gibb and Dr Levin comment on our use of intravenous immunoglobulin in symptomatic HIV infected children, ${ }^{1}$ and quote their experience based on five symptomatic HIV infected children. In the absence of details regarding age, symptoms, and laboratory criteria it is not clear if the five children in their group resemble the HIV infected children we describe in our paper, nor whether their children need any kind of treatment at all.

In addition, in two of their five children, failure to thrive was prominent in the first 18 months of life with spontaneous resolution, and infections were more frequent in the early months of life. In our paper, in only two of the eight children we studied was intravenous immunoglobulin treatment commenced earlier than 24 months, and in all children, strict criteria of at least two or more episodes of bacterial pneumonia, and a three month history of recurrent or chronic upper respiratory sepsis were used. Our children were clinically unwell, and we felt intervention in the form of treatment with intravenous immunoglobulin was justified, and not commenced prematurely.

Regarding the criticism that we used the number of infectious episodes and weight gain as criteria for evaluating intravenous immunoglobulin treatment, we felt that these were consistent and important features of the illness in HIV infected children and that these are key indices of morbidity to evaluate and to influence, if possible. Additionally, we quoted laboratory indices of HIV infection such as thrombocytopenia and the presence of HIV core antigen. There was suppression of the latter in the four children who were antigenaemic and it would be very unexpected for all the antigenaemic children that we studied (who were of different ages) to simultaneously lose their HIV core antigen.

The risk of acquiring hepatitis $\mathrm{C}$ infection from intravenous immunoglobulin is very small and only 43 patients have ever been reported in the world literature. The possibility of increasing HIV activation via antigenic stimulation is unlikely, and we have demonstrated the opposite to occur-that is, a reduction in HIV antigen levels. We do agree that there may be psychological trauma and pain in administering intravenous immunoglobilin to young children. However, there is psychological trauma in repeated hospital admissions for illness, a factor that was reduced with intravenous immunoglobulin treatment.

Finally, we also agree that there have been few reports about the use of intravenous immunoglobulin in HIV infected children. However, the reduction in the incidence of infections and hospital admissions that we observed was of sufficient magnitude for us to recommend a change in clinical practice. The reasons for the difference in opinion put forward by Drs Gibb and Levin may be that although they describe their children as 'symptomatic', their HIV infected children may not in fact suffer as many infections as the HIV infected children we studied.

\section{Breath hydrogen excretion in infants with} colic

SIR,-McKenzie suggests that a shortened intestinal transit time with increased delivery of lactose to the colon might explain our finding of raised excretion of hydrogen in the breath of colicky infants. ${ }^{1}$ Moore, Robb, and Davidson reported that there was no difference in the mean (SD) mouth to caecum transit time between colicky and non-colicky infants $(63.9(37 \cdot 4)$ minutes compared with $58 \cdot 1(43 \cdot 3)$ minutes, respectively). ${ }^{2}$ Raised breath hydrogen excretion in colicky infants may be secondary to the behaviour but a faster transit of lactose in colicky infants does not explain our finding.

McKenzie also refers to the perception that babies with troublesome crying very often stop crying after hospital admission. This is not our experience. We documented the duration of crying and fussing in 46 healthy, 6 week old infants with unsettled behaviour who were admitted to a Tresillian Family Care Centre for a five day residential mothercraft programme. Thirty two (70\%) of these infants still cried and fussed excessively ( $>3$ hours/ day) on the two days before discharge despite intensive mothercraft and paediatric and social worker support.

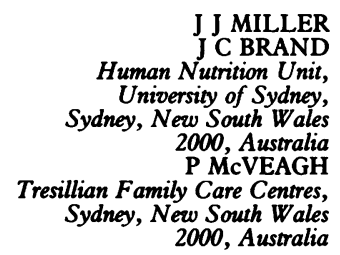

1 McKenzie S. Breath hydrogen excretion in infants with colic. Arch Dis Child 1989;64: 1208 .

2 Moore DJ, Robb TA, Davidson GP. Breath hydrogen response to milk containing lactose hydrogen response to milk containing lactose in colicky and no 